\title{
Identification of DNA and Single Nanoparticles (Qdots) in Muscle Tissue Sections by Low Energy-loss Electron Spectroscopic Imaging
}

\author{
M. Pfannmöller,* I.V. Röder,* G. Benner,** I. Angert,**, I. Wacker,*** and R.R. Schröder* \\ * CellNetworks, Heidelberg University, 69120 Heidelberg, Germany \\ ** Carl Zeiss NTS, 73447 Oberkochen, Germany \\ *** KIT, Karlsruhe Institute of Technology, 76021 Karlsruhe, Germany
}

In electron spectroscopic imaging (ESI) an energy filter allows to selectively use electrons of a defined energy-loss for image recordings. The energy-loss depends on the type of interaction between electrons and sample material. Usually, core losses (inner shell excitations) of several hundred $\mathrm{eV}$ are used to obtain elemental maps of the material investigated [1]. In this way, Cadmium maps were used to image fluorescent nanocrystallites or quantum dots (Qdots) in biological samples [2]. However, spectral information in the low energy-loss range, from approximately 1 to $30 \mathrm{eV}$, comprising inter-band transitions or plasmon excitation losses, can also be used. This was exploited to visualize the fluorescence excitation of doxorubicin in labeled cells at $18 \mathrm{~nm}$ resolution [3].

To improve signal yield for discrimination of different functional materials, many demands on instrumentation, data acquisition and processing techniques have to be met. We used a corrected incolumn energy filter, monochromated beam for high energy resolution and recorded an ESI image series over the entire low-loss range. Resulting stacks of inelastic images were automatically aligned by affine transformations, and processed by multivariate statistical analysis, followed by classification into different spectral groups. Data analysis is incorporated into Ilastik, a software tool dedicated to multispectral data classification [4]. It was shown before that this method is able to identify single Qdots on carbon films [5]. Here we examined sections of resin embedded, highpressure frozen muscle tissue, where Sarcoplasmic/endoplasmic reticulum calcium ATPase was labeled with antibody-conjugated CdTe/ZnS Qdots. DNA (heterochromatin) as well as Qdots can be discriminated from the surrounding environment by means of their spectral, low energy-loss features. Fig. 1a shows a zero-loss filtered image, corresponding inelastic images reveal prominent low-loss signals of DNA (Fig. 1b) and Qdots (Fig. 1c). Using the whole ESI series (from 2 to $28 \mathrm{eV}$ in steps of $1 \mathrm{eV}$ ), a map was created that illustrates the segmentation into background, DNA and Qdots (Fig. 2a). The segmented regions fit perfectly to the structures visible in the zero-loss image (Fig. 2b). Normalized, averaged ESI spectra are given in Fig. 2c, showing a DNA signal at 2 to $8 \mathrm{eV}$ corresponding to UV absorption and a completely different low-loss signal of Qdots compared to background and DNA.

With the method described here we can distinguish material specific signals, visible in low energyloss images and simultaneously identify single functional particles.

References

[1] R.F. Egerton, Rep. Prog. Phys. 72 (2009), 016502.

[2] R. Nisman et al., J. Histochem. Cytochem. 52 (2004), 13.

[3] Mhawi et al., J. Struct. Biol. 158 (2007), 80.

[4] C. Sommer et al., 8th IEEE International Symposium on Biomedical Imaging, ISBI 2011, in press.

[5] M. Pfannmöller et al., in: Proceedings, Microscopy Conference (2009), Graz.

[6] Data were obtained at $60 \mathrm{keV}$ using a Zeiss monochromated Libra 200. 
a

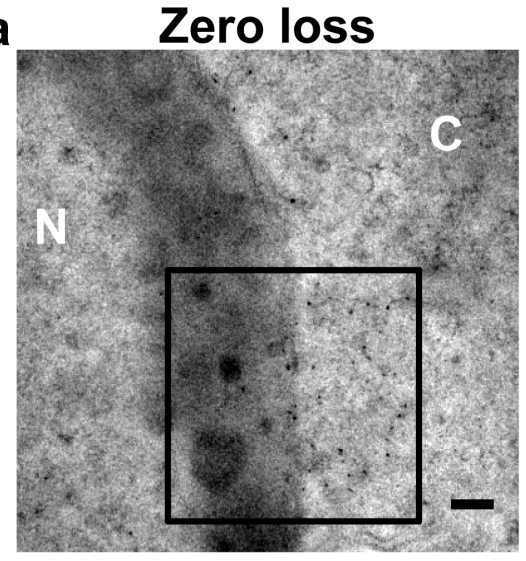

b

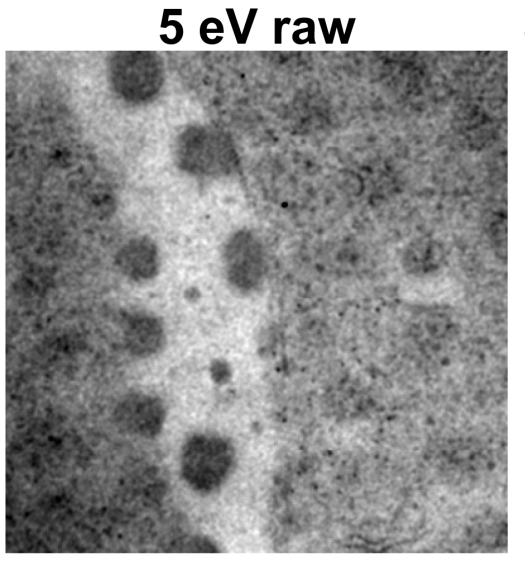

c $10 \mathrm{eV}$ normalized

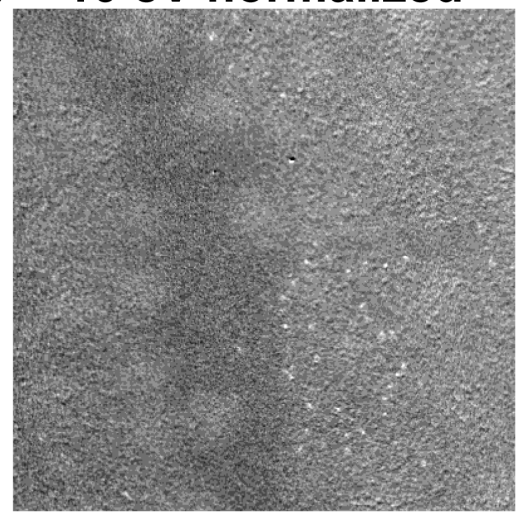

FIG. 1. Images of different energy losses. A zero-loss filtered image (a) showing heterochromatic DNA of a nucleus $(\mathrm{N})$, cytosol (C) of a muscle cell, and several Qdots in the boxed area. In the inelastic image at $5 \mathrm{eV}$ (b) DNA appears bright corresponding to its UV excitation signal. After normalizing the ESI image stack, Qdots appear bright at certain energy losses, e.g. at $10 \mathrm{eV}$ (c). Scale bar in a represents $100 \mathrm{~nm}$.
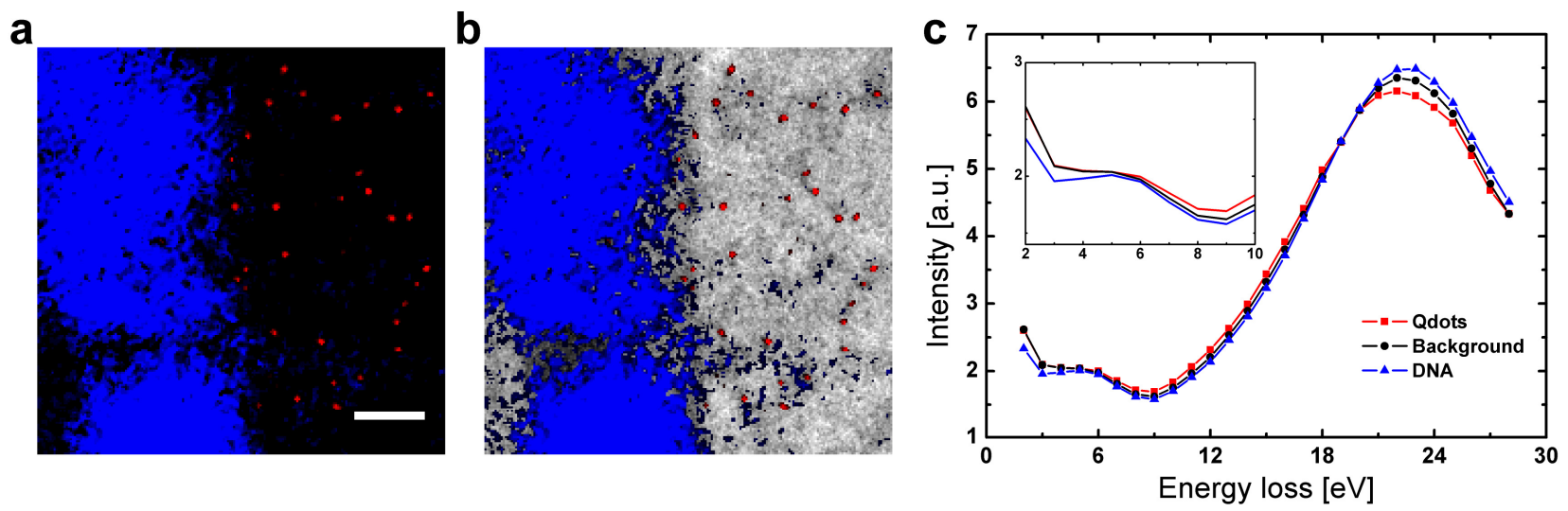

FIG. 2. Segmentation of different objects in the magnified region selected in Fig. 1a according to spectral features by multivariate statistical analysis in Ilastik [4]. In a color map (a) three classes, DNA (blue), Qdots (red), and background signal (black) can be distinguished. The overlay with the zero-loss image (b) reliably identifies single Qdots as well as DNA in the nucleus. Note that here the background is rendered transparent. Averaged, normalized spectra of three classes (c) show spectral differences used by the classification algorithm. Between 3 and $8 \mathrm{eV}$ the DNA signal increases relative to the other spectra (see inset). The Qdot spectrum shows characteristic features, most prominently at plasmon excitation energies. Scale bar in a represents $100 \mathrm{~nm}$. 